Pecvnia, Monográfico (2008), pp. 89-103

\title{
Hacia una nueva perspectiva en dirección de empresas
}

Está ampliamente demostrado que el comportamiento humano no es necesariamente egoísta, pero sin embargo gran parte de las teorías utilizadas en la enseñanza en dirección empresas se basan en esta premisa. El presente artículo invita a la reflexión acerca de las consecuencias que se derivan de esta postura, las dificultades que se presentan a la hora de dar cabida a nuevos planteamientos que promuevan la incorporación de valores más positivos del individuo y la necesidad de que se produzca dicho cambio.

Palabras clave: Conducta egoísta, teoría de la agencia, enseñanza en dirección de empresas.
Roberto Fernández Gago

roberto.fernandez@unileon.es Universidad de León

Organización de Empresas Fac. de Ciencias Económicas y Empresariales Campus de Vegazana, $\mathrm{s} / \mathrm{n}$

24071 León (España)
Key words: Self-interest behaviour, agency theory, teaching of business management. 


\section{INTRODUCCIÓN}

A raíz de los todavía recientes escándalos empresariales, últimamente se observa un profundo ejercicio de reflexión en el mundo académico sobre la consistencia interna de algunas teorías y planteamientos que han conformado la doctrina principal seguida en la enseñanza de dirección de empresas. El artículo de Ghoshal (2005) publicado en el Academy of Management Learning \& Education con el rotundo título "Las malas teorías sobre dirección están destruyendo las buenas prácticas directivas" ha despertado la conciencia de muchos e inspirado a otros tantos. La publicación en esa misma revista de un número $\left(n^{\circ} 3,2006\right)$ dedicado prácticamente en su totalidad a revisar diversos aspectos de la ética empresarial inculcada a los estudiantes de dirección de empresas da buena muestra del movimiento que se está generando y que no debe pasarnos desapercibido.

De acuerdo con los postulados fundamentales de la ciencia económica, todos los agentes económicos están movidos por el egoísmo, es decir, son sujetos maximizadores de funciones de utilidad individuales y poco proclives a tener comportamientos virtuosos per se. Se considera que los mercados de libre competencia garantizan que las acciones individuales en busca del beneficio particular mejoran el bienestar económico del conjunto de la sociedad. Remontándonos a Adam Smith (1776, vc. 1958: 17) nos encontramos con la célebre frase "No es la benevolencia del carnicero, el cervecero o el panadero lo que nos procura nuestra cena, sino la consideración de su propio interés". Actuando así, y conducidos por una mano invisible, se promueve el interés de la sociedad, aunque no fuese esta nuestra intención.

Este planteamiento todavía es defendido con rotundidad por no pocos. La influyente revista Economist (2005: vol. 374, $\mathrm{n}^{\circ} 8410$ ) defiende al capitalismo como la fuerza impulsora del progreso económico y social acaecido durante los últimos 50 años y que aquellos que sospechan del mismo y propugnan la necesidad de una responsabilidad social corporativa lo hacen basándose en dos suposiciones totalmente erróneas: considerar que la búsqueda del beneficio por parte de las empresas no tiene nada que ver con el bien público y dar por sentado que en su loca persecución de la ganancia privada las compañías imponen agobiantes cargas sobre la sociedad y el medio ambiente. 
En cuanto a la economía de los costes de transacción y la teoría de la agencia, han constituido unos cimientos sólidos e imperturbables durante las últimas décadas sobre los que se han construido y desarrollado los principios fundamentales que guían la dirección empresarial y el gobierno corporativo.

La existencia de la empresa ha sido justificada como alternativa a la hora de organizar las transacciones frente al mercado, imponiendo un sistema de jerarquía o autoridad que en ocasiones puede resultar más eficiente que el mecanismo de precios en el que se basan los mercados (Coase 1937, 1960).

Ante la imperfección de los mercados y al no recoger los precios toda la información necesaria para que las partes implicadas en una transacción puedan adoptar una decisión certera acorde con su racionalidad, existe cierto nivel de incertidumbre que puede ser aprovechado por una de ellas para lograr una ventaja individual, es decir, aparece la posibilidad de una conducta oportunista (Williamson 1975). Recogiendo las palabras del propio Maquiavelo, Williamson (1985: 56) declara que "un gobernante prudente no debe cumplir lo que promete cuando ello vaya en contra de sus intereses, ni cuando ya no existan las razones que lo llevaron a comprometerse". Así pues, como se presume que es altamente probable que el ser humano, dada su naturaleza egoísta, intente aprovecharse de esta tentación, habrá que incurrir en unos costes que prevengan la conducta oportunista (información sobre la otra parte, negociación, redacción de contratos y control) o impongan cierta contraprestación si llegara el caso (garantía).

Dados estos costes, tal vez resulte más eficiente coordinar las transacciones dentro de la organización, con un sistema de autoridad en lugar de negociación entre partes. Ahora bien, ¿acaso este sistema no es origen también de situaciones oportunistas generadoras de costes? Lo cierto es que sí, y tanto o más que en el mercado. La estructura establecida para el gobierno de las organizaciones suele descansar sobre el principio de delegación, cediendo una capacidad de decisión que puede ser utilizada de una manera oportunista para conseguir beneficios particulares. Estamos ante la teoría de la agencia (Jensen y Meckling 1976), siendo su máxima expresión la relación establecida al separar propiedad y control entre los accionistas (principales) y los directivos (agentes). Para evitarlo, igualmente habrá que establecer mecanismos (alineación de intereses) e incurrir en costes (control), que en el caso de ser menores que los existentes en el 
mercado determinarían la supremacía de la organización como instrumento de coordinación.

Ante estos planteamientos asumidos de una forma generalizada, al menos en el ámbito académico, cabría realizar algunas reflexiones.

La asunción del Homo Economicus (personas racionales preocupadas únicamente de maximizar su propio interés) no es siempre cierta y responde a una visión lúgubre (gloomy vision) (Hirschman 1970) que lleva a considerar como principal objetivo el de restringir los costes sociales derivados de las imperfecciones humanas. De acuerdo con Sen (1987), la simplificación que se ha hecho de la amplia visión que tenía Adam Smith de los seres humanos es una de las mayores deficiencias de la teoría económica contemporánea, si bien es cierto que él mismo reconoce que:

por más egoísta que se pueda suponer al hombre, evidentemente, existen en su naturaleza algunos principios que le hacen interesarse por la suerte de los otros, de tal modo que la felicidad de éstos le es necesaria, aunque de ella nada obtenga, a no ser el placer de presenciarla (1759, vc. 1997: 49).

En la observación diaria del comportamiento de las personas se reflejan valores como la bondad, benevolencia, nobleza u honor, entre otros. Hay actos como dejar propina en restaurantes que no se visitarán en el futuro, devolver objetos valiosos encontrados en lugares públicos o alistarse como voluntario para participar en un conflicto bélico, los cuales no encajan en absoluto con una perspectiva egoísta del individuo (Frank 2004). Experimentos realizados en este sentido llegan a similares conclusiones. Por ejemplo, en el juego del ultimátum, donde un individuo recibe una cantidad de dinero a repartir con otro obteniendo cada uno su parte si aquél acepta, aunque lo económicamente racional sería ofrecer una cantidad mínima con la tranquilidad de que la oferta será aceptada porque la alternativa es cero, se observa que quien reparte se muestra dispuesto a dar cantidades próximas a la mitad y el que acepta prefiere no recibir nada si la oferta no se considera justa (Guth et al. 1982; Camerer y Thaler 1995).

Por lo tanto, el ser humano no siempre busca su interés más inmediato, sino que habría otras consideraciones adicionales que no se están considerando con la misma intensidad. En numerosas ocasiones, hay un comportamiento cooperativo capaz de generar confianza y que es 
difícil de explicar por los postulados económicos. Una justificación podría provenir de la teoría de la selección de especies, válida para la cooperación entre individuos relacionados genéticamente (Hamilton 1964). Otro argumento posible es la persecución de beneficios propios basados en relaciones de largo plazo (Trevers 1971; Axelrod 1981; Nowak et al. 1995) o su búsqueda mediante compensaciones indirectas y el desarrollo de una reputación (Leimar y Hammerstein 2001; Gintis et al. 2001). Y a pesar de todas estas posibles explicaciones, aún existen situaciones donde las personas se comportan cooperativamente aunque no haya relación entre ellas, no sean repetitivas y aunque la posibilidad de sacar rédito mediante la mejora de su reputación sea mínima o nula (Fehr y Gächter 2002).

Esta otra realidad no parece estar recogida suficientemente en los modelos económicos, siempre preocupados por la vertiente negativa e imperfecta del ser humano. La mayoría del valor no se crea a través de transacciones individuales en el mercado, sino por organizaciones en las que la gente actúa colectiva y cooperativamente, con sus motivaciones y acciones coordinadas y guiadas por un propósito común, con sus defectos, pero también con sus virtudes. Los modelos tradicionales, en lugar de centrarse en el proceso de creación de valor, se preocupan en exceso por su apropiación y la posibilidad de que alguien se aproveche indebida y oportunistamente en dicho reparto. Esto conduce en ocasiones a la sumisión y obediencia, al control institucional como tarea esencial del directivo frente a una visión más positiva que facilite la cooperación y valore la iniciativa, donde difundir la confianza y liderar el cambio sean las verdaderas funciones directivas (Ghoshal et al. 1999).

Admitiendo otras cualidades del ser humano cambiarían drásticamente nuestras teorías (Ghoshal 2005).

\section{EL INMOVILISMO Y SUS EFECTOS}

Si es cierto que existen estos errores, o al menos limitaciones, en los supuestos básicos de las teorías existentes, cabe preguntarse por qué motivo se vienen mantenido de una forma continuada en el tiempo.

Ghoshal (2005) sostiene que detrás de la investigación y desarrollo de la dirección de empresas hay un trasfondo ideológico que dificulta su evolución por caminos distintos. Esta ideología, que parte del individualismo radical y liberalismo, ha sido promovida por la Escuela de 
Chicago, siendo Milton Friedman uno de sus principales exponentes (Friedman 2002).

Por otro lado, puede existir una justificación desde el lado de la demanda (Kanter 2005). El enfoque basado en los intereses de los accionistas e incentivos financieros, como propone la teoría de la agencia, se puso de moda en los años 80 en Estados Unidos al haber una audiencia deseosa de teorías que evitaran que las empresas permanecieran cautivas de sus directivos. Aunque hubo quien promulgó una visión más amplia considerando otros stakeholders, no resultó una postura bien recibida en un momento en el que el mundo había rechazado rotundamente el socialismo en cualquier vertiente frente al capitalismo. Lo que debía primar era el interés individual $y$, más concretamente, el de los accionistas. Las enseñanzas en dirección de empresas debían centrarse en que esta premisa fuese siempre respetada.

Existe otro motivo altamente plausible para mantener la atención centrada en un objetivo de maximización de valor o beneficio y es la mayor facilidad a la hora de plantear modelos y contrastar hipótesis (Ghoshal 2005; Kanter 2005). En el caso de considerar otras motivaciones y otros stakeholders distintos a los accionistas, seguramente nos acercaríamos más a la realidad pero dificultaría su concreción práctica. No obstante, medir el éxito exclusivamente con datos económicos resulta una pobre aproximación de lo que es el bienestar personal (Frey y Stutzer 2002) y no considera el impacto provocado en el entorno ambiental (Dunlap y Van Liere 1984).

Por último, puede haber cierto rechazo desde los propios académicos o resistencia al cambio. Al esfuerzo adicional que esta adaptación supone, se une el hecho de que resulta difícil publicar ideas innovadoras en este sentido. Ya existen voces diversas que proclaman las limitaciones de una visión excesivamente centrada en la riqueza de los accionistas y las presunciones negativas de la teoría de la agencia, pero se encuentran con dificultades para ser escuchadas incluso en el propio mundo académico (Waddock 2006).

Esta postura e inmovilismo conllevan una serie de consecuencias no deseadas sobre las que conviene reflexionar.

En primer lugar, y en términos de eficacia, hay que señalar que las prácticas derivadas de la teoría de la agencia con el objeto de maximizar el valor para los accionistas, tales como la retribución directiva mediante stock options, aumento de consejeros externos y separación de 
funciones entre presidente y CEO o mercados de control con la posibilidad de opas hostiles, no han tenido los efectos previstos (Ghoshal 2005). Esto quiere decir que tomando como válidas las presunciones anteriormente mencionadas sobre la naturaleza e intereses del ser humano, ni siquiera se ha conseguido solucionar correctamente el problema planteado. Sin embargo, y a pesar de la falta de respaldo empírico, la teoría de la agencia continúa dominando la investigación académica sobre gobierno corporativo (Daily et al. 2003).

Por otro lado, dada la naturaleza social de la ciencia económica, se corre el riesgo de que las teorías influyan en los fenómenos estudiados y esta influencia puede ser tal que lleve a convertirlas en verdaderas. Los directivos actúan de acuerdo con las teorías sobre management existentes y a la vez son protagonistas de la realidad que es estudiada en dichas teorías, con lo que existe un riesgo claro de manipulación. Concretamente, el oportunismo o propensión a comportarse oportunistamente de un individuo, supuesto básico en la teoría de la agencia, se ve influido por la valoración que haga de la otra parte con la que realiza la transacción, sea individuo, grupo u organización (Ajzen y Fishbein 1977; Eagly y Chaiken 1992) y por unos condicionantes previos como son sus actitudes y valores, creados a través de su exposición, consciente y subliminalmente, a diversos estímulos (Krosnic et al. 1992).

Centrados en el primero de los dos tipos de influencia mencionados, es cierto que los mecanismos de gobierno jerárquico aumentan los costes del comportamiento oportunista y en ese sentido disminuyen la probabilidad de que ocurran, pero por otra parte perjudican el sentimiento hacia la entidad, lo que puede traducirse en una mayor propensión a comportarse oportunistamente. Por lo tanto, el resultado neto dependerá de la relación entre estos dos efectos. De acuerdo con la evidencia parece que el efecto neto es el contrario al previsto por Williamson (Ghoshal y Moran 1996).

En cuanto a los condicionantes previos como valores y actitudes del individuo, se verán afectados por la educación y formación que haya recibido. En este sentido, es posible plantearse si las enseñanzas en dirección de empresas están condicionando la propensión a comportarse de una manera oportunista, primando siempre el interés propio (Donaldson 2002). Los estudios existentes parecen concluir que efectivamente es así. Hay evidencia (Ferraro et al. 2005) de que los alumnos con formación en economía son más propensos a asumir un comportamiento de free-rider (p.ej. Marwell y Ames 1981), a no colaborar al encontrarse en situaciones 
del tipo dilema del prisionero (p.ej. Frank et al. 1993), a comportarse más egoístamente en juegos de ultimátum (Carter y Irons 1991; Kahneman et al. 1986 a y b) o a sucumbir a la tentación de obtener una ganancia privada, aunque haya que actuar deshonestamente (Frank y Schulze 2000).

Por otro lado, también se ha demostrado que estos alumnos son más proclives a hacer trampas para conseguir mejores calificaciones con menor esfuerzo y consideran esta conducta menos rechazable que sus compañeros de otras titulaciones (Baird 1980; Bowers 1964; McCabe y Treviño 1995; McCabe 1997; McCabe et al. 2006).

Es posible que los estudiantes con tendencia a comportarse egoístamente y buscar su propio beneficio a toda costa, incluso haciendo trampas si es necesario, actúen así por una motivación intrínseca como puede ser el deseo de acumular dinero y que todo ello sea previo al comienzo de sus estudios, eligiendo precisamente los estudios de dirección de empresas porque entienden que les ayudarán a alcanzar sus objetivos. Hay evidencia empírica que acredita que en ocasiones esto no es así. Frank et al. (1993) demostraron que los estudiantes que cursaron un curso de economía, expuestos al modelo tradicional de búsqueda del interés propio, se comportaban más egoístamente que otros que siguieron uno de astronomía. En la misma línea, el Aspen Institute (2003) desveló un cambio en los valores de los estudiantes tras cursar un programa MBA, pasando a importar más el valor obtenido por los accionistas como medida del éxito empresarial que las necesidades de los consumidores y la calidad del producto.

Incluso en el caso de que existan unos atributos personales que sean los condicionantes básicos de la conducta, el código ético bajo el que se actúa en el mundo de los negocios es importante y debe enseñarse. Aunque siempre habrá ejemplos de abuso, una sociedad que glorifica tales abusos sufrirá las peores consecuencias (Gapper 2005).

\section{UNA NUEVA PERSPECTIVA}

Según Samuelson (2006), ha llegado el momento de tomar una decisión: continuar como si la perspectiva y planteamientos que se vienen utilizando en la enseñanza de la dirección de empresas fuese suficiente para afrontar los retos actuales o crear una oportunidad para que los estudiantes reflexionen sobre el futuro de los negocios como 
poderoso agente de cambio y fuerza social. Giacalone y Thompson (2006) sostienen que ayudar a los estudiantes a ser más responsables socialmente y sensibles éticamente es una parte sustancial de la responsabilidad de los docentes, apostando por una visión más humanista del mundo cuyos valores principales resalten no sólo la importancia de los resultados financieros. Mientras prestigiosas escuelas de negocios como la Harvard Business School propugnan como misión "formar líderes que cambien el mundo" (www.hbs.edu/about/justwhatis.html), es difícil recordar un tiempo en el que la educación en dirección de empresas haya estado sometida a un escrutinio tan severo o haya sido tan criticada como actualmente.

Una alternativa a la visión del directivo comportándose de forma egoísta y emocionalmente desligado de su compañía es la proporcionada por la teoría stewardship (Davis et al. 1997 y 2004). Basada en la psicología y sociología, surgió con el propósito de estudiar aquellas situaciones en las que los ejecutivos actuaban como administradores motivados por la defensa de los intereses corporativos. Ahora las conductas a favor del colectivo y de la organización tienen una utilidad mayor que las individualistas y centradas en uno mismo. Si este es su pensamiento, comportarse de acuerdo al mismo y no egoístamente no supone una falta de racionalidad.

No obstante, también hay que reconocer que antes de la teoría de la agencia ya existían ejemplos de prácticas directivas indeseables motivadas por el interés propio. Esta evidencia no permite ser tan optimista sobre la naturaleza humana como para pensar que el modelo stewardship funcionaría solo y Hambrick (2005) propone que el verdadero reto consiste en fusionar lo mejor del modelo stewardship y del de la agencia.

Otra vertiente distinta se corresponde con la corriente de pensamiento denominada "Positive Organizational Scholarship" (Cameron et al. 2003), que se ocupa del estudio de los atributos, procesos y efectos especialmente positivos de las organizaciones y sus miembros. En esta línea, Cameron (2006) reclama la necesidad de incluir en el contenido de los programas formativos de dirección de empresas las características que definen el virtuosismo, tales como el honor, la bondad, la benevolencia o la nobleza y no fijarse sólo en el lado negativo del espectro. Los valores morales o principios que evitan el daño no son los mismos que los que llevan a hacer el bien. El virtuosismo produce energía positiva en los sistemas, permite el crecimiento y construye capital social, lo que a su vez desarrolla el capital intelectual y propicia ventajas organizativas (Nahapiet y Ghoshal 1998). 


\section{DISCUSIÓN}

Después de numerosos escándalos empresariales, a las escuelas de negocios se les ha empezado a considerar responsables de crear líderes éticos. Según Ghoshal (2005), las teorías económicas y la filosofía del libre mercado que conforman los cimientos de gran parte del currículo en las escuelas de negocios han tenido un impacto nocivo sobre los valores, actitudes y comportamiento de los estudiantes. La mayoría de los economistas estarían de acuerdo en que la gente a menudo se preocupa de algo más que de su propio bienestar material y también tiene en consideración el bienestar de los demás. Sin embargo, pocos economistas incluyen esta perspectiva más amplia en sus modelos de comportamiento humano (Frank 2004).

Por un lado, como defensa al egoísmo y oportunismo de los directivos, en el campo de la dirección habitualmente se enfatiza la maximización de la riqueza para el accionista sin prestar igual atención a otros stakeholders. Dentro del paraguas de la denominada responsabilidad social corporativa se están recogiendo nuevas prácticas directivas que adoptan una visión más amplia y dotan de mayor trascendencia al papel de la empresa en la sociedad. Pero su origen no debe buscarse en las aulas, sino en la realidad económica y social, pues la enseñanza en dirección de empresas parece ir por detrás de los cambios en vez de provocarlos. Es más, el retardo con el que muchas veces se incorporan los fenómenos observados en la práctica real del mundo de los negocios a las doctrinas es injustificadamente largo.

Pero además, la responsabilidad social corporativa sigue respondiendo a la versión tradicional de comportamiento ético que se resiste a incorporar valores adicionales al simple cumplimiento de obligaciones. Por "responsabilidad" se entiende el cargo u obligación moral que resulta del posible yerro en un asunto determinado. De nuevo se trata de evitar el daño que un comportamiento egoísta puede estar generando a los demás, sólo que en este caso al pensar en los demás no se hace únicamente referencia a los accionistas.

La propensión a cooperar y otras conductas humanas en ocasiones no son más que la respuesta a una serie de valores tan intrínsecos al individuo como lo puede ser el egoísmo y, sin embargo, no parecen merecer la misma atención. 
Dado el impacto que las teorías y principios económicos tienen en la sociedad por influir sensiblemente en las decisiones de dirigentes empresariales y políticos, hay quien manifiesta la necesidad de reconsiderar detenidamente si la inercia que empuja la investigación y docencia en dirección de empresas es la correcta. Sin duda, cualquier cambio al respecto exige reflexión, pero también convencimiento y valentía. Tal vez las teorías precedentes promuevan su autocumplimiento, desanimando a adoptar posturas marginales (Ghoshal 1996). Quizás por los nuevos caminos que se abren sea más complicado elaborar elegantes modelos con proposiciones claramente contrastables y prescribir o aconsejar prácticas directivas simples y determinantes (Kanter 2005). Es seguro que este esfuerzo naciente es poco reconocido en cuanto a potenciales publicaciones y por tanto puede ser perjudicial para la carrera académica y profesional de quien lo asuma (Waddock 2006). No obstante, según Giacalone y Thompson (2006), parece una necesidad, obligación y responsabilidad correr dicho riesgo con el fin último de cambiar la tendencia de los últimos 50 años y ofrecer un panorama más amplio y enriquecedor de la dirección de empresas.

\section{BIBLIOGRAFÍA}

AJZEN, I. \& M. FISHBIEN (1977) "Attitude-Behavior Relations: A Theoretical Analysis and Review of Empirical Research", Psychological Bulletin, 84 (5), pp. 888-918.

ASPEN INSTITUTE (2003) "Where Will They Lead: MBA Student Attitudes About Business and Society. Disponible en: http://www.aspeninstitute. org/site/apps/ka/ec/searchit.asp?c=huLWJeMRKpH\&b=667387\&en=hrKT LTPvEcIRLWOvE8IHJQPzEkKZJ3PxF9KRJ9PILkK1Kgl (Accedido el 15/01/07).

AXELROD, R. \& W.D. HAMILTON (1981) "The Evolution of Cooperation", Science, 211, pp. 1390-1396.

BaIRD, J.S. (1980) "Current Trends in College Cheating", Psychology in the Schools, 17 (4), pp. 515-522.

Bowers, W.J. (1964) Student Dishonesty and its Control in College. New York, NY: Bureau of Applied Social Research, Columbia University. 
Camerer, C. \& R.H. Thaler (1995) "Ultimatums, Dictators and Manners", Journal of Economic Perspectives, 9 (2), pp. 209-219.

CAMERON, K. (2006) "Good or Not Bad: Standards and Ethics in Managing Change", Academy of Management Learning \& Education, 5 (3), pp. 317-323.

CAMERON, K.S.; J.E. DutTON \& R.E. QUINn (Eds.) (2003) Positive Organizational Scholarship. San Francisco, CA: Berret-Koehler.

CARTER, J.R. \& M.D. IRONS (1991) "Are Economists Different, and if so, why?", Journal of Economic Perspectives, 5 (2), pp. 171-177.

COASE, R.H. (1960) "The Problem of Social Cost", Journal of Law and Economics, 3 (1), pp. 1-44.

- (1937) "The Nature of the Firm", Economica, 4 (16), pp. 386-405.

DAILY, C.M.; D.R. DALTON \& A.A.Jr. CANNELLA (2003) "Corporate Governance: Decades of Dialogue and Data", Academy of Management Review, 28 (3), pp. 371-382.

DAVIS, J.H.; F.D. SCHOORMAN \& L. DONALDSON (2004) "Toward a Stewardship Theory of Management". T. CLARKE (Ed.) Theories of Corporate Governance. Oxon: Routledge, pp. 118-134.

- (1997) "Toward a Stewardship Theory of Management", Academy of Management Review, 22 (1), pp. 20-47.

DONALDSON, L. (2002) "Damned by Our Own Theories: Contradictions Between Theories and Management Education", Academy of Management Learning and Education, 1 (1), pp. 96-106.

DUNLAP, R.E. \& K.D. VAN LIERE (1984) "Commitment to the Dominant Social Paradigm and Concern for Environmental Quality", Social Science Quarterly, 65 (4), pp. 1013-1028.

Eagly, A.H. \& S. Chalken (1992) The Psychology of Attitudes. San Diego, CA: Harcourt Brace Jovanovich.

FEHER, E. \& S. GÄCHTER (2002) "Altruistic Punishment in Humans", Nature, 415 (6868), pp. 137-140.

Ferraro, F.; J. Pfeffer \& R.I. Sutton (2005) "Economics Language and Assumptions: How Theories Can Become Self-Fulfilling", Academy of Management Review, 30 (1), pp. 8-24.

Frank, B. \& G.G. SCHULze (2000) "Does Economics Make Citizens Corrupt?", Journal of Economic Behavior and Organization, 43 (1), pp. 101-113.

Frank, R.H. (2004) What Price the Moral High Ground?. Princeton, NJ: Princeton University Press. 
-, T. GILOVICH \& D.T. Regan (1993) "Does Studying Economics Inhibit Cooperation?", Journal of Economic Perspectives, 7 (2), pp. 159-171.

FREY, B.S. \& A. STUTZER (2002) "What Can Economists Learn from Happiness Research?", Journal of Economic Literature, 40 (2), pp. 402-435.

FRIEDMAN, M. (2002) Capitalism and Freedom, Ed. 40 aniversario. Chicago, IL.: The University of Chicago Press.

GAPPER, J. (2005) "Comment on Sumantra Ghoshal's 'Bad Management Theories Are Destroying Good Management Practices"', Academy of Management Learning \& Education, 4 (1), pp. 101-103.

GHosHAL, S. (2005) "Bad Management Theories Are Destroying Good Management Practices", Academy of Management Learning \& Education, 4 (1), pp. 75-91.

- \& P. MORAN (1996) "Bad for Practice: A Critique of the Transaction Cost Theory", Academy of Management Review, 21 (1), pp. 13-47.

-, C. BARTLETT \& P. MORAN (1999) "A New Manifesto for Management", Sloan Management Review, 40 (3), pp. 9-20.

GIACAlone, R.A. \& K.R. THOMPSON (2006) "Business Ethics and Social Responsibility Education: Shifting the Worldview", Academy of Management Learning \& Education, 5 (3), pp. 266-277.

GINTIS, H.; E. SMITH \& S. BOWLES (2001) "Costly Signalling and Cooperation", Journal of Theoretical Biology, 213 (1), pp. 103-119.

Guth, W.; R. SChmittberger \& B. Schwarze (1982) "An Experimental Analysis of Ultimatum Bargaining", Journal of Economic Behavior and Organization, 3 (4), pp. 367-388.

HAMBRICK, D.C. (2005) "Just How Bad Are our Theories? A Response to Ghoshal", Academy of Management Learning \& Education, 4 (1), pp. 104-107.

Hamilton, W.D. (1964) "The Genetical Evolution of Social Behavior I and II", Journal of Theoretical Biology, 7 (1), pp. 1-52.

HIRSCHMAN, A.O. (1970) "The Search for Paradigms as a Hindrance to Understanding", World Politics, 22 (3), pp. 329-343.

JENSEN, M. \& W. MECKLING (1976) "Theory of the Firm: Managerial Behaviour, Agency Costs and Ownership Structure", Journal of Financial Economics, 3 (4), pp. 305-360.

KAHNEMAN, D.; J. KNETSCH \& R. ThALER (1986a) "Fairness and the Assumptions of Economics", Journal of Business, 59 (4), pp. 285-300. 
,$--\&-(1986 b)$ "Fairness as a Constraint on Profit Seeking: Entitlements in the Market Perceptions of Unfairness: Constraints on Wealth Seeking", American Economic Review, 76 (4), pp. 728-741.

KANTER, R.M. (2005) "What Theories do Audiences Want? Exploring the Demand Side", Academy of Management Learning \& Education, 4 (1), pp. 93-95.

KROSNIC, J.A.; A.L. BETZ, L.J. JUSSIM \& A.R. LYNN (1992) "Subliminal Conditioning of Attitudes", Personality and Social Psychology Bulletin, 18 (2), pp. 152-162.

LEIMAR, O. \& P. HAMMERSTEIN (2001) "Evolution of Cooperation through Indirect Reciprocity", Proceedings of the Royal Society of London: Biological Sciences, 268, pp. 745-753.

MARWELL, G. \& R.E. AMES (1981) "Economists Free Ride, Does Anyone Else?", Journal of Public Economics, 15 (3), pp. 295-310.

MCCABE, D.L. (1997) "Classroom Cheating Among Natural Science and Engineering Majors", Science \& Engineering Ethics, 3 (4), pp. 433-445.

- \& L.K. TREVIÑo (1995) "Cheating Among Business Students: A Challenge for Business Leaders and Educators", The Journal of Management Education, 19 (2), pp. 205-218.

-, K.D. ButTERFIELD \& L.K. TREVIÑo (2006) "Academia Dishonesty in Graduate Business Programs: Prevalence, Causes, and Proposed Action", Academy of Management Learning \& Education, 5 (3), pp. 294-305.

NAHAPIET, J. \& S. GHOSHAL (1998) "Social Capital, Intellectual Capital, and the Organizational Advantage", Academy of Management Review, 23 (2), pp. 242-266.

NOWAK, M.A.; R.M. MAY \& K. SIGMUND (1995) "The Arithmetics of Mutual Help", Scientific American, 272 (6), pp. 76-81.

Samuelson, J. (2006) "The New Rigor: Beyond the Right Answer", Academy of Management Learning \& Education, 5 (3), pp. 356-365.

SEN, A. (1987) On Ethics and Economics. Oxford: Basil Blackwell.

SMITH, A. (1776) The Wealth of Nations. Londres. [vc. (1958): Investigación sobre la naturaleza y causas de la riqueza de las naciones. México: Fondo de Cultura Económica].

- (1759) The Theory of Moral Sentiments, $1^{\text {a }}$ Ed. Londres. [(1790): The Theory of Moral Sentiments, 6a Ed.] [vc. (1997): La teoría de los sentimientos morales. Madrid: Alianza]. 
TRIVERS, R. (1971) "The Evolution of Reciprocal Altruism", Quarterly Review of Biology, 46 (1), pp. 35-47.

WADDOCK, S. (2006) "Forging a Path for Ethics and Business in Society", Academy of Management Learning \& Education, 5 (3), pp. 334-345.

WILLIAMSON, O.E. (1985) The Economic Institutions of Capitalism. New York, NY: Free Press.

- (1975) Markets and Hierarchies: Analysis and Antitrust Implications. New York, NY: Free Press. 